This document is the Accepted Manuscript version of the following article: Amira Guirguis, Sarah Girotto, Benedetta Berti, and Jacqueline L. Stair, 'Identification of new psychoactive substances (NPS) using handheld Raman Spectroscopy employing both 785 and 1064 nm laser sources', Forensic Science International, published online 4 February 2017, doi:

http://dx.doi.org/10.1016/j.forsciint.2017.01.027. Under embargo. Embargo end date: 4 February 2018.

This manuscript version is made available under the CC-BY-NC-ND 4.0 license

http://creativecommons.org/licenses/by-nc-nd/4.0/

(c) 2017 Elsevier Ltd. All rights reserved. 
Identification of new psychoactive substances (NPS) using handheld Raman Spectroscopy employing both 785 and 1064 nm laser sources.

\author{
Amira Guirguis ${ }^{\mathrm{a}}$ (a.guirguis2@herts.ac.uk) \\ Sarah Girotto $^{\mathrm{a}}$ (sara.girotto90@gmail.com) \\ Benedetta Bertia (benedetta.berti@hotmail.it) \\ and Jacqueline L. Stair*a
}

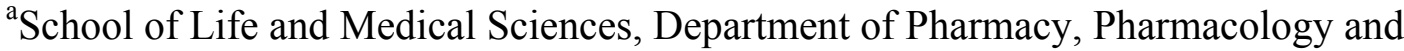
Postgraduate Medicine, University of Hertfordshire, Hatfield, UK

\title{
Correspond to:
}

Dr. Jacqueline L. Stair

Department of Pharmacy,

Pharmacology and Postgraduate Medicine

University of Hertfordshire

College Lane,

Hatfield, AL10 9AB, UK.

Email: j.stair@herts.ac.uk

Tel: +44 (0)1707 284000 Ext: 3367

Fax: +44 (0)1707 284870 


\begin{abstract}
The chemical identification of new psychoactive substances (NPS) in the field is challenging due not only to the plethora of substances available, but also as a result of the chemical complexity of products and the chemical similarity of NPS analogues. In this study, handheld Raman spectroscopy and the use of two excitation wavelengths, 785 and $1064 \mathrm{~nm}$, were evaluated for the identification of 60 NPS products. The products contained a range of NPS from classes including the aminoindanes, arylalkylamines, benzodiazepines, and piperidines $\&$ pyrrolidines. Identification was initially assessed using the instruments' in built algorithm (i.e., \% HQI) and then further by visual inspection of the Raman spectra. Confirmatory analysis was preformed using gas chromatography mass spectrometry. For the 60 diverse products, an NPS was successfully identified via the algorithm in 11 products (18\%) using the $785 \mathrm{~nm}$ source and 29 products $(48 \%)$ using the $1064 \mathrm{~nm}$ source. Evaluation of the Raman spectra showed that increasing the excitation wavelength from 785 to $1064 \mathrm{~nm}$ improved this 'first pass' identification primarily due to a significant reduction in fluorescence, which increased $\mathrm{S} / \mathrm{N}$ of the characteristic peaks of the substance identified. True positive correlations between internet products and NPS signatures ranged from 57.0 to $91.3 \%$ HQI with typical RSDs $<10 \%$. Tablet formulations and branded products were particularly challenging as a result of low NPS concentration and high chemical complexity, respectively. This study demonstrates the advantage of using a $1064 \mathrm{~nm}$ source with handheld Raman spectroscopy for improved 'first pass' NPS identification when minimal spectral processing is required, such as when working in field. Future investigations will focus on the use of mixture algorithms, effect of NPS concentration, and further improvement of spectral libraries.
\end{abstract}




\section{Introduction}

In recent years, the use of new psychoactive substances (NPS) has proliferated globally. ${ }^{[1]}$ NPS, also known as 'legal highs', designer drugs, 'herbal highs', 'bath salts' and 'research chemicals', are often perceived as 'legal' synthetic recreational drugs with analogous pharmacological effects to internationally controlled drugs of abuse. ${ }^{[2]}$ The UK Psychoactive Substances Act 2016, recently enacted, defines these substances as 'capable of producing a psychoactive effect in a person who consumes it'. ${ }^{\text {[3] }}$ The United Nations Office on Drugs and Crimes (UNODC) stated that up to December 2013, over 445 NPS had been reported worldwide. In Europe alone, approximately two NPS were notified weekly to the Early Warning System (EWS) in 2014. ${ }^{[4]}$ NPS include newly invented compounds (e.g., STS-135), revisited research chemicals (e.g., MT-45) ${ }^{[4,5]}$ diverted pharmaceuticals (e.g., gabapentin), products marketed as food supplements (e.g., adrafinil), and resurged controlled drugs of abuse (e.g., carfentanyl, an analogue of the opioid fentanyl ${ }^{[4]}$ Hence, the term 'new' was employed to indicate 'newly misused' and 'newly synthesised' in clandestine laboratories rather than simply 'newly invented'. ${ }^{[6]}$

The abuse of NPS can result in varied and unpredictable harm as there are no regulations concerning content, potency, point of sale and purchase age. ${ }^{[7]}$ This is compounded by the facile dissemination via free movement across borders, head shops and easy internet sales. They are deceitfully marketed to imply legality and safety ${ }^{[2]}$ and often branded with attractive names such as pink champagnes and pink panthers. The abuse of NPS has been linked to violence and aggression ${ }^{[8]}$, sympathomimetic symptoms ${ }^{[9]}$,acute organ failure ${ }^{[10]}$ psychosis $^{[9]}$ as well as fatalities. ${ }^{[11]}$ At present, the dearth of pharmacological and toxicological knowledge on NPS increases the potential risks and harms to users and greatly impacts treatment decisions. ${ }^{[12]}$ The net contribution of NPS abuse to adverse health consequences and crimes is still unknown as they are not easily detected using common forensic and toxicology screening tests. ${ }^{[13]}$ Thus, the sheer number and chemical variety of NPS along with their potential health risks impacts both health and legal authorities. ${ }^{[2]}$ As a result, countries such as the UK are taking measures to control these substances, ${ }^{[3]}$ where chemical monitoring will be key to achieving this.

The identification and characterisation of NPS to inform risk assessment and drug control pose a great analytical challenge. This is again due to the sheer number of NPS, the permeations of mixtures that can be concocted with and without adulterants, and the continued emergence of new (i.e., unknown) chemical substances. According to the UNODC, gas chromatography - mass spectrometry (electron ionisation) (GC-MS (EI)) was the predominant analytical technique employed by EU countries for chemical analysis of NPS mixtures. ${ }^{[1]}$ For example, this technique has been shown to successfully discriminate between NPS classes ${ }^{[14-17]}$ as well as within classes such as cathinones, ${ }^{[18]}$ aminoindanes ${ }^{[19,20]}$ and benzylpiperazines. ${ }^{[21]}$ In circumstances where no reference standards or data were available, nuclear magnetic resonance spectroscopy (NMR) has been used to determine the chemical connectivity of NPS such as cathinones ${ }^{[22]}$ and aminoindanes. ${ }^{[19,20]}$ High performance liquid chromatography (HPLC) was used with various detectors to separate compounds between 
NPS classes ${ }^{[16]}$ as well as within classes of cathinones ${ }^{[23]}$ and cannabinoids. ${ }^{[24]}$ In general, the techniques mentioned above require transport to a forensic laboratory and solvent dissolution/filtering before analysis. In contrast, vibrational techniques such as Fourier transform infrared (FT-IR), near infrared (NIR), and Raman spectroscopy can perform rapid analysis in the solid-state and are available in handheld and portable versions, which are advantageous for in-field testing for law enforcement and healthcare professionals. ${ }^{[25]}$ FT-IR has traditionally been a preferred forensic technique due its selectivity and specificity. ${ }^{[18]}$ For example, FT-IR was used to discriminate between three different trifluoromethylmethcathinone analogues ${ }^{[18]}$ and two methylenedioxypyrovalerone (MDPV) isomers. ${ }^{[26]}$ Although FT-IR can be used in the solid-state, analysis through packaging is often difficult and interferences from excipients/cutting agents are common. The use of NIR spectroscopy for the identification of NPS also shows promise, ${ }^{[27,28]}$ but NIR is more susceptible to moisture effects, physical properties and cutting agents, and often requires careful selection of data treatment. The use of Raman spectroscopy may overcome these challenges as it offers a number of advantages for in-field testing such as high discrimination power, ${ }^{[29]}$ minimal to no sample preparation, through package analysis, and low sensitivity to moisture, physical properties ${ }^{[30]}$ and cutting agents. ${ }^{[31]}$ A recent study evaluated the use of hand-held FT-IR, NIR and Raman spectrometers for NPS analysis, where Raman spectroscopy preformed the best when identifying the components in model mixtures. ${ }^{[31]}$

Although the UNODC does not report on the use of Raman spectroscopy for NPS characterisation, ${ }^{[1]}$ it is considered a Category A forensic technique. ${ }^{[29]}$ For that reason, Raman analysis has been employed in research and forensic analysis ${ }^{[32]}$ for the characterisation of drugs of abuse such as 3,4-methylenedioxy-N-methylamphetamine (MDMA), cocaine and heroin. ${ }^{[33]}$ More recently, a number of studies have evaluated the use of Raman for NPS products. ${ }^{[32,34-38]}$ Maheux and Copland (2011) used a range of analytical techniques including Raman spectroscopy for the identification of cathinones in seized samples. ${ }^{[34]}$ Studies also reported on the use of Raman spectroscopy to discriminate between cathinone regioisomers ${ }^{[35]}$ and derivatives ${ }^{[32]}$ using benchtop Raman instruments employing a laser excitation wavelength $\left(\lambda_{\text {ex }}\right)$ of $785 \mathrm{~nm}$. Bell and coworkers recently reported on the use of Raman spectroscopy for the identification of a range of NPS products using an $\lambda_{\text {ex }}$ of 785 nm. ${ }^{[38]}$ From these studies, a challenge when using Raman to analyse NPS 'street samples' was fluorescence, often resulting from impurities and/or cutting agents, which can mask the signal from the active ingredients in the product. ${ }^{[31,39,40]}$ Goodacre and coworkers investigated the use of surface enhanced Raman spectroscopy (SERS) to enhance the Raman signal while also reducing interference due to fluorescence. ${ }^{[36,37]}$ Although SERS is a viable approach for fluorescence reduction, careful and invasive sample preparation is often needed. An alternative approach which requires no sample preparation is the use of a longer $\lambda_{\text {ex }}$ (e.g., $1064 \mathrm{~nm}$ ) which has been shown to improve identification of traditional drugs of abuse such as cocaine and amphetamine. ${ }^{[40,41]}$ At present there remains limited information on the use of hand-held Raman spectroscopy for the wide range of NPS products available and the feasibility to improve NPS identification by using a longer $\lambda_{\text {ex }}$. 
The aim of this study was to investigate a wide range of NPS products purchased from the internet using handheld Raman spectroscopy and to evaluate the performance of two wavelengths, 785 and $1064 \mathrm{~nm}$, for the identification of these substances using a 'first pass' identification algorithm and further assessment of the Raman spectra.

\section{Experimental}

\subsection{Chemicals and Reagents}

The reference standards of eight NPS drugs, eight adulterants and twelve cutting agents were used for the study. The NPS reference standards (Figure 1) 2-aminoindane (2-AI), 5,6-methylenedioxy-2-aminoindane (MDAI), 1-benzofuran-5-ylpropan-2-amine (5-APB), 1-benzofuran-6ylpropan-2-amine (6-APB), 1-(thiophen-2-yl)-2methylamino propane (MPA), etizolam and methylphenidate (MPD) were purchased from LGC standards (Teddington, UK); and dextromethorphan (DXM) was purchased from Sigma Aldrich (Dorset, UK). The adulterants benzocaine (BEN), caffeine (CAF), lidocaine (LID), paracetamol (PAR), phenacetin (PHE) and theophylline (THEO) were purchased from Sigma Aldrich (Dorset, UK); diltiazem (DIL) was obtained from the Medicines Testing Lab (UK); and procaine (PRO) was obtained from British Drug Houses (London, UK). The cutting agents calcium carbonate $\left(\mathrm{CaCO}_{3}\right)$, creatine (CRE), dextrose (DEX), glucose (GLU), lactose (LAC), L-tyrosine (L-TYR), magnesium stearate (MGS), microcrystalline cellulose (MCC), niacinamide (NIA), sucrose (SUC), talc (TAL) and taurine (TAU) were purchased from Sigma Aldrich (Dorset, UK). Sixty NPS products (i.e., powders, capsules and tablets) were purchased from the internet (Tables 13), under a Home Office licence, and selected according to their label claim and UNODC classification. Additional details for the 60 NPS are provided in Table S1 (Supplementary Information). Powders and capsules were emptied into clear glass vials (Kimble Chase vial screw thread with PTFE cap, China) for Raman analysis, while the tablets were crushed using an agate mortar and pestle before transferring into glass vials. The glass vials were vortex mixed before collection of each spectrum using a VORTEX-GENIE2 (Scientific industries, Inc., USA) mixer for $30 \mathrm{~s}$, shaken, then the process repeated. For GC-MS analysis, solutions (1 mg $\mathrm{mL}^{-1}$ ) of each standard and product was prepared in HPLC grade methanol from Fisher Scientific (Loughborough, UK), except for the benzodiazepine tablets which were concentrated to ca. $45 \mathrm{mg} \mathrm{mL}^{-1}$ and filtered through 0.2 $\mu \mathrm{m}$ PTFE membrane filters (National Scientific Company, USA) prior to

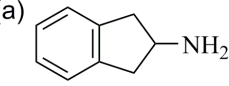

(b)

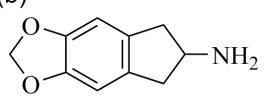

(c)

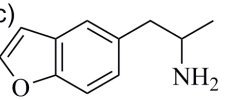

(d)

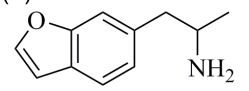

(e) $\mathrm{H}$
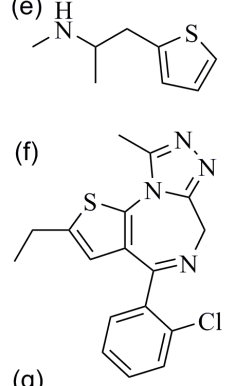

(g)
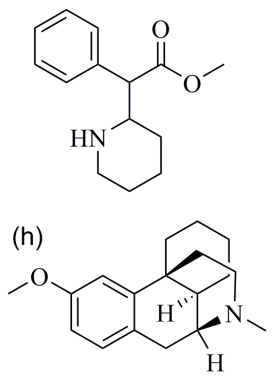

Fig. 1: The chemical structures of (a) 2-AI, (b) 5,6MDAI, (c) 5APB, (d) 6-APB, (e) MPA, (f) Etizolam (g) MPD and (h) DXM. analysis. 


\subsection{Analysis of NPS and related substances using handheld Raman spectroscopy}

Two handheld Raman instruments with different laser sources, Xantus-1 and First-Guard (Rigaku, USA), were employed for the analysis of NPS products. Specifications of both instruments are shown in Table S2 (Supplementary Information). Four methods were used to collect the Raman spectra depending on the nature of the substance and included method A (1000 ms exposure time; $300 \mathrm{~mW}$ laser power; 2 accumulations), method B (5000 ms exposure time; $490 \mathrm{~mW}$ laser power; 2 accumulations), method C (500 ms exposure time; $200 \mathrm{~mW}$ laser power; 2 accumulations), and method D (1000 ms exposure time; $100 \mathrm{~mW}-1$ $\mathrm{mW}$ laser power; 2 - 25 accumulations). All samples were initially run using method A, but method B was used for samples that displayed a poor Raman signal. Method C was used for samples that were burned from long exposure time and/or high laser power (i.e., coloured samples or samples containing fluorescing chemicals). Method D was developed in an attempt to collect Raman signals from challenging samples, which exhibited very high fluorescence background and/or burned with method $\mathrm{C}$. This was done by adopting an iterative approach to reducing the laser power and increasing the number of accumulations. All methods used baseline correction, and the dark background was corrected for every 15 minutes. The instruments were calibrated each day before analysis using a benzonitrile reference standard (Rigaku, US). Most reference standards and products were analysed directly through glass vials after optimisation of the vial holder attachment with respect to the focal point. For NPS standards that were limited in quantity, $2 \mathrm{mg}$ were placed on aluminium foil plates (Fisher Scientific, China) and the signal was optimised using the adjustable probe tip. All substances were analysed in triplicate. Raman spectra of the reference substances ( $\mathrm{n}=$ 28) were added to the on-board factory spectral library, which was composed of 260+ spectra of common chemical substances. For a 'first pass' identification, the spectra from the NPS products were automatically compared to the on-board reference library and reported a percentage hit quality index (\% HQI) correlation. The mean \pm the standard deviation of the highest hit was calculated from the triplicate measurements and reported. If the correlations between the triplicate analyses were inconsistent, this was reported instead of a mean value. The product spectra were also visually inspected and compared to reference spectra, which was also used to evaluate the findings of the matching algorithm.

\subsection{Confirmatory analysis of NPS and related substances using gas chromatography - mass spectrometry (GC-MS)}

GC-MS analysis was used to confirm the identity of compounds present in the purchased internet products. The analysis was performed using a Varian 240 ion trap MS equipped with a Varian 450 gas chromatography instrument and a Varian 8400 auto-sampler from Agilent Technologies (Berkshire, UK). Samples were analysed using electron ionization (EI) with a scan range of m/z 40 - 1000. An Agilent Technologies column (30 m x $0.25 \mathrm{~mm}$ x $0.25 \mu \mathrm{m})$ coated with a $0.50 \mathrm{~mm}$ film of $50 \%$ phenyl $-50 \%$ methyl polysiloxane was used with helium gas as the mobile phase at a flow rate of $1 \mathrm{ml} \mathrm{min}^{-1}$. A CP-1177 injector was held at $275{ }^{\circ} \mathrm{C}$ and was used in split mode (10:1) for most samples, but in splitless mode when low signals were observed. An injection volume of $1 \mu \mathrm{L}$ was used for all samples. The column temperature was programmed as follows: $50{ }^{\circ} \mathrm{C}$ for $2 \mathrm{~min}$, ramped to $300{ }^{\circ} \mathrm{C}, 15{ }^{\circ} \mathrm{C} \mathrm{min}{ }^{-1}$, 
held for $5 \mathrm{~min}$, then cooled to $50{ }^{\circ} \mathrm{C}$; the total run time was $28.67 \mathrm{~min}$. The mass spectra obtained were compared to the purchased reference standards and the following EI spectral libraries: NIST (Version v. 1.0.2.2), SWGDRUG MS (Version 2.1 (2014)) and Cayman (Version v. 04292014).

\section{Results and Discussion}

\subsection{In-built identification method (or instrument algorithm)}

Raman responses were initially evaluated using a 'first pass' identification algorithm, percentage hit quality index (\% HQI), which is used to measure the similarity between the measured spectrum of an unknown material against library signatures of known references. ${ }^{[42]}$ HQI is calculated as shown in Eqn (1) and indicates how much the test spectrum correlates to the library signature(s) in \%. Unlike raw material identification where the \% HQI is commonly set between 80 and $99 \%$, NPS products may contain mixtures of NPS, adulterants, and cutting agents, which can result in a lower \% HQI, yet still correlating to the most prominent substance. ${ }^{[42]}$ The extent of this change in relation to the composition will be reviewed. Evaluation of the Raman spectra was carried out and compared to the results of the matching algorithm for the NPS reference standards and products.

$$
\mathrm{HQI}=\frac{(\text { library } . \text { unknown })^{2} * 100}{(\text { library } \cdot \text { library })(\text { unknown } . \text { unknown })} \quad(\text { Eqn 1) }
$$

\subsection{Raman analysis of purchased reference standards}

A number of pure substances (i.e., eight NPS, eight adulterants and 12 cutting agents) were purchased as reference standards based on the NPS purchased from the internet. A spectrum was collected for each substance and stored in the on-board library. A representative spectrum for each NPS reference standard using the $1064 \mathrm{~nm}$ source is provided in Supplementary Information (Figure S1). When comparing the spectra visually, they all have distinctive Raman fingerprints, except for 5 and 6-APB. In this case, the spectra are difficult to distinguish, as the chemical structures differ only in the substitution position of the alkylamine. In the case of the aminoindane analogues, 2-AI and 5,6-MDAI, the chemical structures differ by a methylenedioxy group (Figure 1). Addition of this moiety to the 2aminoindane backbone resulted in the appearance of two distinct peaks at 713 and $1350 \mathrm{~cm}^{-1}$ likely due to the $-\mathrm{C}=\mathrm{C}$ - cis-di-substituted deformation vibrations and methoxy stretching vibration. ${ }^{[43]}$ The spectra for etizolam and MPA each have only one predominant peak at 1496 and $1438 \mathrm{~cm}^{-1}$ respectively, which may effect the identification of these substances. Although MPA, 5-APB, and 6-APB fall under the category of arylalkylamines, the spectrum of MPA shows clear differences to the APB isomers' spectra. MPD, a piperidine, showed characteristics peaks 996, 1189, 1431, 1587 and $1723 \mathrm{~cm}^{-1}$. DXM from the 'other category' showed characteristic peaks at $686,853,1245$, and $1439 \mathrm{~cm}^{-1}$. To test the accuracy and selectivity of each instruments' algorithm, the standards were run as test samples (NPS standard results are presented in Table S3 Supplementary Information). Using the $785 \mathrm{~nm}$ 
source, 27 out of 28 standards were consistent with their library signature with \% HQI values ranging from $90 \pm 10$ to $100 \%$. Microcrystalline cellulose resulted in inconsistent correlations to lactose and amylose from potato. The spectra indicated that the mismatch was likely the result of a high fluorescent background with minimal peaks seen for all three replicates. When using the longer $1064 \mathrm{~nm}$ wavelength, 27 out of 28 standards correlated to their library signature. Of those, 23 standards were consistent with their library signature with $\%$ HQIs ranging from $90.0 \pm 0.9$ to $100.0 \pm 0.1 \%$. Four samples correlated with \% HQIs < $90 \%$ which were 2-AI, 5,6-MDAI, 6-APB and MPA with \% HQI ranging from $72 \pm 1$ to 85 \pm 1 . The only mismatch for the $1064 \mathrm{~nm}$ instrument was for magnesium stearate (MGS). The MGS spectrum correlated to the signature of beeswax, a chemically similar compound, as the first hit (90 $\pm 4 \%$ HQI), but correlated to MGS in all measurements as the second hit $(80 \pm 2$ $\%$ HQI). In summary, both instruments showed selectivity for the majority of standards run as test samples, including the APB positional isomers. Fluorescence affected one sample using the $785 \mathrm{~nm}$, and poor Raman scatterers often gave slightly reduced \% HQIs (i.e., 70 $90 \%$ ) for the $1064 \mathrm{~nm}$. Slight reductions in \% HQI for these particular standards were perhaps also a consequence of needing to run these standards on Al plates with a small sample size. Although an optimisation protocol was followed, variations in the beamwidth and distance to the target substance can influence spectral quality effecting the reproducibility and \% HQI value during validation but also when analysing NPS products.

\subsection{Raman and GC-MS analysis of NPS internet products}

The effect of using different $\lambda_{\text {ex }}$ (i.e., 785 and $1064 \mathrm{~nm}$ ) for the identification of NPS internet products was assessed. In this study, 60 NPS products were analysed using two handheld Raman instruments using a 'first pass' in-built matching algorithm and evaluation of the Raman spectra. The NPS products analysed covered a wide range of categories according to the European Monitoring Centre for Drugs and Drug Addiction (EMCDDA) classification ${ }^{[44]}$ (Table S1, Supplementary Information). GC-MS was employed to confirm the identity of volatile/semi-volatile compounds present in the NPS products.

\subsubsection{Aminoindanes}

Twelve aminoindane samples, purchased from the internet, were analysed using both Raman instruments and GC-MS (Table 1). Internet products included three of the most popular aminoindane substances, 2-AI, 5-IAI and MDAI. ${ }^{[4]}$ Aminoindanes are amphetamine analogues and have been shown to be potent serotonin-releasing substances. ${ }^{[45]}$ The GC-MS results indicated that 11 out of the 12 products did contain an aminoindane, ${ }^{[20,46]}$ while only nine products contained the aminoindane reported on the label claim. When using the standard $785 \mathrm{~nm}$ laser, only four of the NPS products (i.e., P6, 8, 9 and 12) correlated to an aminoindane substance using the algorithm. These four products correlated to 5,6-MDAI, as confirmed by GC-MS, with \% HQIs ranging from $60 \pm 8$ to $84 \pm 10$. A high fluorescent background and low Raman signal were observed for P1, 2, 4, 10 and 11. These products correlated to MPA at \% HQIs ranging from $96.3 \pm 0.8$ to $97.0 \pm 0.3$, but MPA was not confirmed using GC-MS except in P11. This was likely the result of the MPA standard spectrum (i.e., the spectral library signature) displaying a high fluorescent background 
(Figure S2 in Supplementary Information). Consequently, the MPA signature correlated highly to NPS product spectra with similar backgrounds and little to no Raman bands, resulting in the false positives. For example, the Raman spectrum of P11 (Figure 2a) showed small peaks at ca. 714, 782 and $864 \mathrm{~cm}^{-1}$, which match Raman bands for 5,6-MDAI, but due to the large fluorescent background this sample correlated to MPA at $96.5 \pm 0.2 \%$. As the Raman bands for MPA at $1442 \mathrm{~cm}^{-1}$ were not visible, this correlation was likely also a false positive. Product 5 and 7 showed some higher intensity Raman peaks on a fluorescent background but resulted in no correlations (no match). This may have occurred as the HQI algorithm considers fluorescence signals as additional characteristics of the unknown sample. ${ }^{[47]}$ Interestingly, whilst P3 was confirmed to contain both 5,6-MDAI and CAF, the spectra collected using the $785 \mathrm{~nm}$ source correlated to CAF demonstrating the challenge of identifying NPS in a complex mixture adulterated with a relatively strong Raman scatterer. The 12 samples were then analysed using the $1064 \mathrm{~nm}$ source where 9 of the 12 samples (P1, 2,5-9,11 and 12) correlated to the NPS present in the sample with HQIs ranging from $60 \pm$ 6 to $91.3 \pm 0.4 \%$. When using the $1064 \mathrm{~nm}$ instrument, fluorescence was significantly reduced for many of the products as shown for P11 (Figure 2b). This resulted in improved spectral definition and subsequent identification. Figure $3 \mathrm{a}$ shows a closer look for two examples, 2-AI (P1) and 5,6-MDAI (P11), comparing the NPS reference and product spectra. Although the GC-MS data indicated that both 2-AI and 5,6-MDAI were present in P1, the Raman peaks consistent with 2-AI (i.e., at 775, 844, 1020, 1205, and $1236 \mathrm{~cm}^{-1}$ ) dominate the spectrum. P11 was also confirmed to contain two NPS, MPA and 5,6-MDAI, where most peaks were consistent with 5,6-MDAI such as 713 and $1355 \mathrm{~cm}^{-1}$, however, peaks for MPA were also visible at 1038 an $1433 \mathrm{~cm}^{-1}$. It is important to note that two products, P4 and P11, contained a combination of MDAI and MPA, which has been reported to have synergistically and/or additive effects. ${ }^{[48]}$ In summary, the use of a lower energy wavelength reduced fluorescence, which improved signal to noise of discriminating peaks and 'first pass' identification of the active NPS for 5 of the 12 aminoindane internet products, resulting in a total of 9 products with a correctly identified NPS. 
Table 1: Results from the analysis of aminoindane and arylalkylamine internet products using two handheld Raman spectrometers $\left(\lambda_{\mathrm{ex}}=785\right.$ and $\left.1064 \mathrm{~nm}\right)$ and GC-MS ${ }^{1}$

\begin{tabular}{|c|c|c|c|c|c|c|c|c|}
\hline \multirow[t]{2}{*}{$\begin{array}{c}\text { Product } \\
\text { No. }\end{array}$} & \multirow[t]{2}{*}{ Product name } & \multicolumn{2}{|c|}{$\begin{array}{c}\text { Handheld Raman } \\
785 \mathrm{~nm} \\
\end{array}$} & \multicolumn{2}{|c|}{$\begin{array}{c}\text { Handheld Raman } \\
1064 \mathrm{~nm} \\
\end{array}$} & \multicolumn{3}{|c|}{ GC-MS } \\
\hline & & ID & $\% \mathrm{HQI}$ & ID & $\% \mathrm{HQI}$ & $\begin{array}{l}\text { RT } \\
(\min )\end{array}$ & $\begin{array}{l}\text { Base } \\
\text { Peak } \\
(\mathbf{m} / \mathbf{z}) \\
\end{array}$ & MS ID \\
\hline \multicolumn{9}{|c|}{ Aminoindanes } \\
\hline 1 & 2-AI & $\mathrm{MPA}^{3}$ & $96.6 \pm 0.4$ & $2-\mathrm{AI}$ & $80 \pm 4$ & $\begin{array}{l}9.9 \\
12.6\end{array}$ & $\begin{array}{l}133 \\
160\end{array}$ & $\begin{array}{l}\text { 2-AI } \\
5,6-\mathrm{MDAI}\end{array}$ \\
\hline 2 & $2-\mathrm{AI}$ & $\mathrm{MPA}^{3}$ & $96.3 \pm 0.8$ & 2-AI & $91.3 \pm 0.4$ & 9.8 & 133 & $2-\mathrm{AI}$ \\
\hline 3 & 5-IAI & CAF & $87 \pm 2$ & CAF & $80 \pm 10$ & $\begin{array}{l}12.6 \\
14.4\end{array}$ & $\begin{array}{l}160 \\
194\end{array}$ & $\begin{array}{l}\text { 5,6-MDAI } \\
\text { CAF }\end{array}$ \\
\hline 4 & 5-IAI & $\mathrm{MPA}^{3}$ & $96 \pm 0.7$ & $\begin{array}{l}\text { Benzyl } \\
\text { Alcohol }\end{array}$ & $80 \pm 2$ & $\begin{array}{l}9.0 \\
9.4 \\
12.6 \\
14.64\end{array}$ & $\begin{array}{l}58 \\
133 \\
160 \\
86\end{array}$ & $\begin{array}{l}\text { MPA } \\
2-\mathrm{AI} \\
5,6-\mathrm{MDAI} \\
\mathrm{NC}\end{array}$ \\
\hline 5 & MDAI & No match & & 5,6-MDAI & $60 \pm 6$ & 12.8 & 160 & 5,6-MDAI \\
\hline 6 & MDAI & 5,6-MDAI & $84 \pm 10$ & 5,6-MDAI & $80.3 \pm 0.2$ & 12.8 & 160 & 5,6-MDAI \\
\hline 7 & MDAI & No match & & 5,6-MDAI & $80 \pm 2$ & 12.7 & 160 & 5,6-MDAI \\
\hline 8 & MDAI & 5,6-MDAI & $60 \pm 8$ & 5,6-MDAI & $80.7 \pm 0.1$ & 12.8 & 160 & 5,6-MDAI \\
\hline 9 & MDAI & 5,6-MDAI & $75 \pm 6$ & 5,6-MDAI & $80.1 \pm 0.5$ & 12.9 & 160 & 5,6-MDAI \\
\hline 10 & MDAI & $\mathrm{MPA}^{3}$ & $97.0 \pm 0.3$ & No match & & $\begin{array}{l}14.0 \\
14.4 \\
15.2 \\
15.5\end{array}$ & $\begin{array}{l}192 \\
191 \\
206 \\
177\end{array}$ & $\begin{array}{l}\mathrm{NC} \\
\mathrm{NC} \\
\mathrm{NC} \\
\mathrm{NC}\end{array}$ \\
\hline 11 & MDAI & $\mathrm{MPA}^{3}$ & $96.5 \pm 0.2$ & 5,6-MDAI & $64.0 \pm 1.8$ & $\begin{array}{l}8.7 \\
12.6\end{array}$ & $\begin{array}{l}58 \\
160\end{array}$ & $\begin{array}{l}\text { MPA } \\
5,6-\mathrm{MDAI}\end{array}$ \\
\hline 12 & MDAI & 5,6-MDAI & $67 \pm 4$ & 5,6-MDAI & $80 \pm 2$ & 12.8 & 160 & 5,6-MDAI \\
\hline \multicolumn{9}{|c|}{ Arylalkylamines } \\
\hline 13 & APB & $\mathrm{MPA}^{3}$ & $95.2 \pm 0.4$ & 5-APB & $80 \pm 2$ & $\begin{array}{l}11.6 \\
16.4\end{array}$ & $\begin{array}{l}44 \\
126\end{array}$ & $\begin{array}{l}\text { 5-APB } \\
\text { Pyrovalerone }^{2}\end{array}$ \\
\hline 14 & 5-APB & $\mathrm{MPA}^{3}$ & $97.2 \pm 0.6$ & 5-APB & $55.0 \pm 0.6$ & 11.6 & 44 & $5-\mathrm{APB}$ \\
\hline 15 & 5-APB & $\mathrm{MPA}^{3}$ & $94 \pm 1$ & $5-\mathrm{APB}$ & $60 \pm 1$ & 11.4 & 44 & 5-APB \\
\hline 16 & 5-APB & $\mathrm{MPA}^{3}$ & $95.8 \pm 0.7$ & No match & & 11.7 & 44 & 5-APB \\
\hline 17 & $5-\mathrm{APB}$ & $\mathrm{MPA}^{3}$ & $95.1 \pm 0.1$ & Data acquisi & on failed & $\begin{array}{l}11.8 \\
12.3\end{array}$ & $\begin{array}{l}44 \\
134\end{array}$ & $\begin{array}{l}5-\mathrm{APB} \\
5-\mathrm{APDB}^{2}\end{array}$ \\
\hline 18 & 6-APB & \multicolumn{2}{|c|}{ Inconsistent correlations } & $\mathrm{MCC}$ & $77.4 \pm 0.2$ & 11.6 & 44 & $5-\mathrm{APB}$ \\
\hline 19 & 6-APB & \multicolumn{2}{|c|}{ Inconsistent correlations } & 6-APB & $50 \pm 3$ & $\begin{array}{l}11.4 \\
11.7 \\
12.9\end{array}$ & $\begin{array}{l}44 \\
44 \\
160\end{array}$ & $\begin{array}{l}\text { 5-APB } \\
6 \text {-APB } \\
5,6-\mathrm{MDAI}\end{array}$ \\
\hline 20 & 5-MAPB & \multicolumn{2}{|c|}{ Inconsistent correlations } & 5-APB & $60 \pm 4$ & 12.1 & 58 & $5-\mathrm{MAPB}^{2}$ \\
\hline 21 & MPA & $\mathrm{MPA}^{3}$ & $94 \pm 2$ & MPA & $82.9 \pm 0.7$ & $\begin{array}{l}9.1 \\
16.6\end{array}$ & $\begin{array}{l}58 \\
271\end{array}$ & $\begin{array}{l}\text { MPA } \\
\text { DXM }\end{array}$ \\
\hline 22 & MPA & $\mathrm{MPA}^{3}$ & $98.1 \pm 0.2$ & MPA & $60 \pm 2$ & 9.2 & 58 & MPA \\
\hline 23 & MPA & $\mathrm{MPA}^{3}$ & $97.2 \pm 0.1$ & MPA & $78.2 \pm 0.8$ & $\begin{array}{l}8.8 \\
11.9 \\
12.0 \\
14.4 \\
17.4\end{array}$ & $\begin{array}{l}58 \\
58 \\
44 \\
194 \\
110\end{array}$ & $\begin{array}{l}\text { MPA } \\
\text { NC } \\
6-A P B \\
\text { CAF } \\
\text { 5-MeO-DALT }\end{array}$ \\
\hline 24 & MPA & $\mathrm{MPA}^{3}$ & $98.1 \pm 0.2$ & MPA & $81.2 \pm 0.8$ & 8.8 & 58 & MPA \\
\hline 25 & MPA & $\mathrm{MPA}^{3}$ & $96 \pm 1$ & MPA & $80 \pm 2$ & 8.7 & 58 & MPA \\
\hline 26 & MPA & $\mathrm{MPA}^{3}$ & $96 \pm 3$ & MPA & $80 \pm 2$ & 8.8 & 58 & MPA \\
\hline
\end{tabular}


(a)

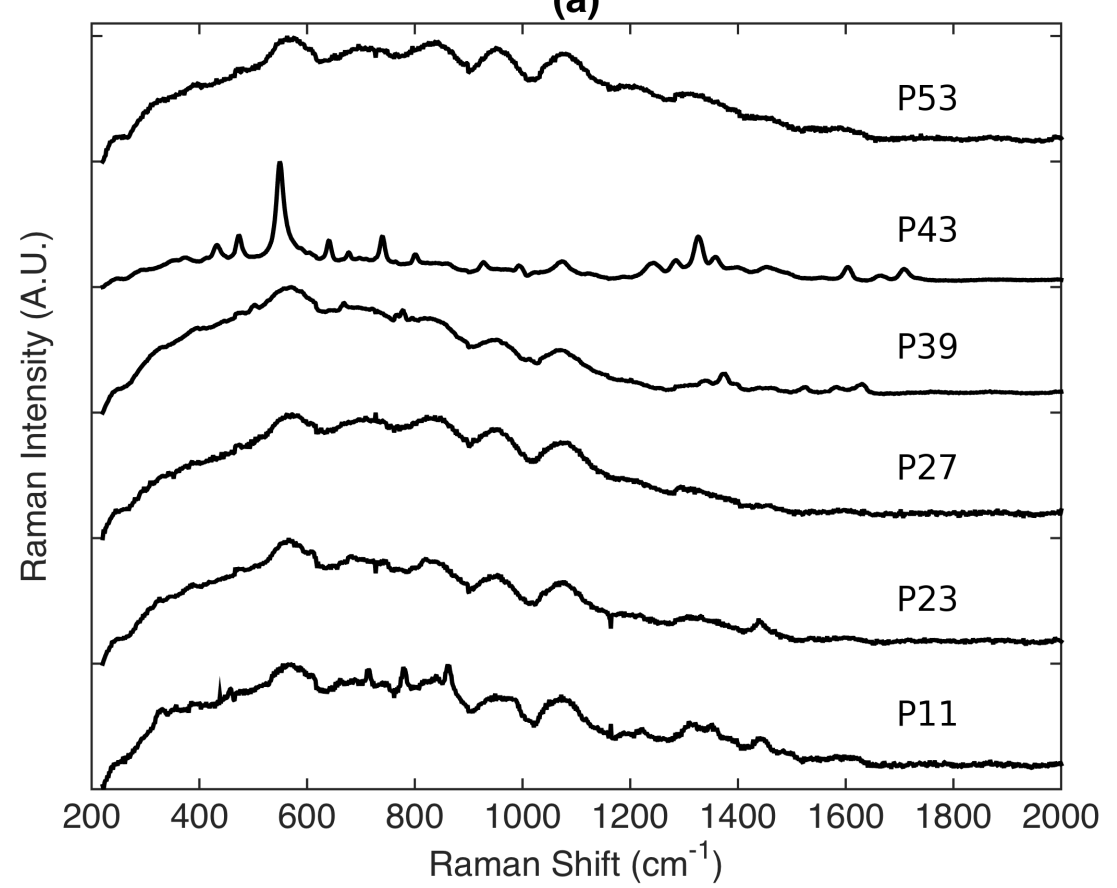

(b)

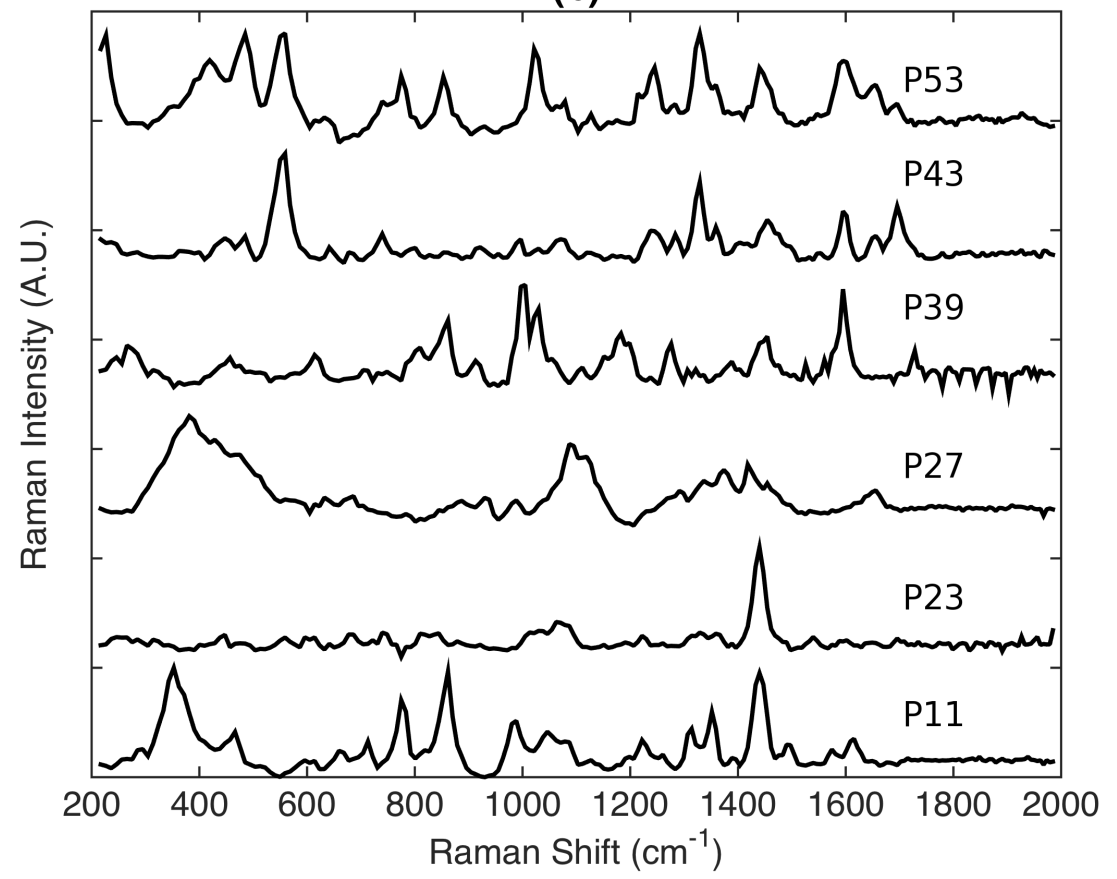

Fig. 2: Raman spectra of selected NPS internet products using the $785 \mathrm{~nm}$ (a) and $1064 \mathrm{~nm}$ (b) excitation wavelength. The products analysed were P11, P23, P27, P39, P43, and P53 with the label claim of MDAI, MPA, Etizolam, EPD, DXM, and Pink Champagnes, respectively, which does not necessarily represent the sample composition. The spectra presented have been normalised to the maximum peak. 


\subsubsection{Arylalkylamines}

Fourteen arylalkylamines samples, purchased from the internet, were analysed using both Raman instruments and GC-MS (Table 1). These included the aminopropylbenzofurans (APB) isomer/analogues 5-APB, 6-APB and 1-(Benzofuran-5-yl)- $N$-methylpropan-2-amine (5-MAPB). Methyl aminopropamine (MPA) products, reported to exert stimulant effects, ${ }^{[48]}$ were also investigated. The GC-MS results showed that all 14 products contained an arylalkylamine, ${ }^{[46,49]}$ while 13 products contained the arylalkylamine reported on the label claim. When using the standard $785 \mathrm{~nm}$ laser, most samples (i.e., P13 - 17 and P21 - 26) correlated to MPA (94 \pm 2 to $98.1 \pm 0.2 \% \mathrm{HQI})$ while three (i.e., P18 - 20) resulted in inconsistent correlations. Upon investigation of the spectra (e.g., see Figure 2 a for spectra of P23), all samples showed a high degree of fluorescence with little to no distinct Raman bands indicating false positive correlations to the MPA signature. Therefore, after visual inspection it was suggested that no true positive correlations to an arylalkylamine were found in any of the 14 products using the $785 \mathrm{~nm}$ source. The 14 products were then analysed using the 1064 $\mathrm{nm}$ source where 10 products (i.e., P13-15, 19, 21-26) correlated to the NPS present in the sample with HQIs ranging from $50 \pm 3$ to $82.9 \pm 0.7 \%$. Again, a reduction in fluorescence improved signal to noise of peaks and thus 'first pass' identification of the NPS samples. The spectra from the NPS that correlated to MPA using an $\lambda_{\text {ex }}$ of $1064 \mathrm{~nm}$ showed a distinct peak around $1442 \mathrm{~cm}^{-1}$ with no fluorescence indicting true positive correlations (e.g., see Figure $2 \mathrm{~b}$ for spectra of P23). Figure $3 \mathrm{~b}$ compares the reference and product spectra for two examples, MPA (P23) and 5-APB (P13). As mentioned previously, MPA has only one strong peak with other minor peaks, which has been useful for the identification of P23 in this case as no other notable peaks are visible. For the Raman spectrum of P13 most peaks could be attributed to 5-APB (i.e., 758, 1258, 1326, and $1530 \mathrm{~cm}^{-1}$ ); the spectrum correlated to 5-APB at $80 \pm 2 \%$ HQI. In regards to the APB analogues, their Raman spectra are very similar as they only differ in the substitution position of the alkylamine, however a key discriminating peak can be observed at $1350 \mathrm{~cm}^{-1}$ for 6-APB and then slight peaks shifts seen at ca. 1110, 1430 and $1600 \mathrm{~cm}^{-1}$ (Figure S1 in Supplementary Information). There was a correlation to MCC (77.4 $\pm 0.2 \%$ ) for P18 even though 5-APB was present in the sample, this suggests that the cutting agent concentration was in a higher proportion compared to the active ingredient. ${ }^{[31]}$ In the case of P20, a $60 \pm 4 \%$ correlation to 5-APB was found as no 5-MAPB signature was present in the Raman library. This demonstrates that a substance may be correlated to a similar structural analogue using the algorithm, which can assist with identifying suspect NPS. Samples P16 and 17 resulted in 'no match' and failed data acquisition due to sample burning, even when using low power, as they were both of a dark colour. ${ }^{[30]}$ The use of a lower energy laser wavelength reduced fluorescence from both the cutting agents present and coloured samples improving 'first pass' identification of the NPS ingredient for 11 of 14 arylalkylamines products. 
(a)

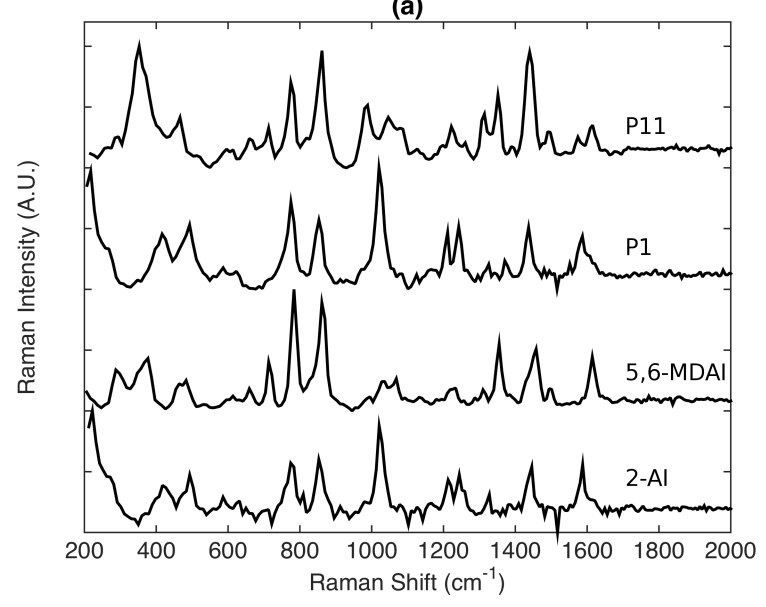

(c)

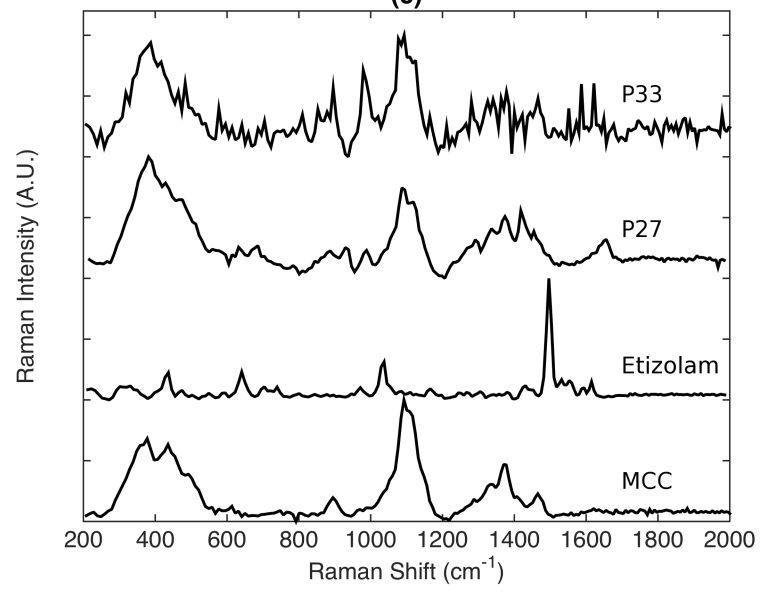

(e)

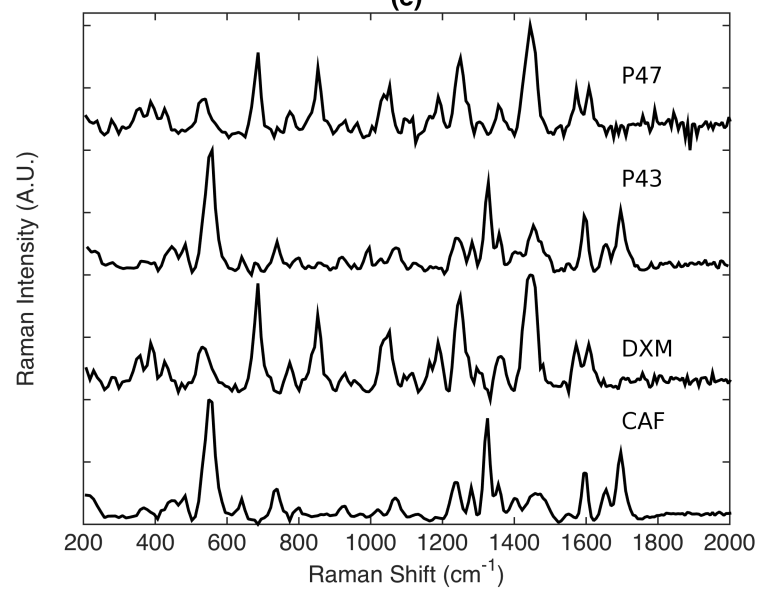

(b)

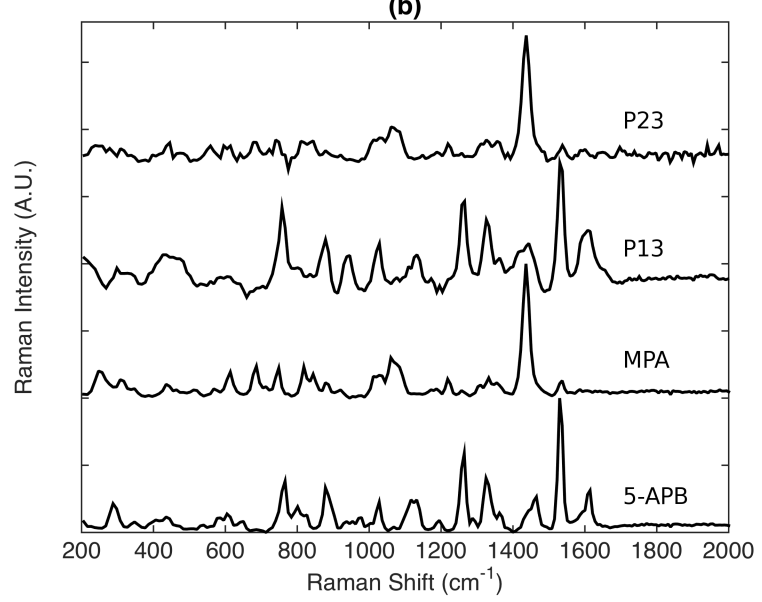

(d)

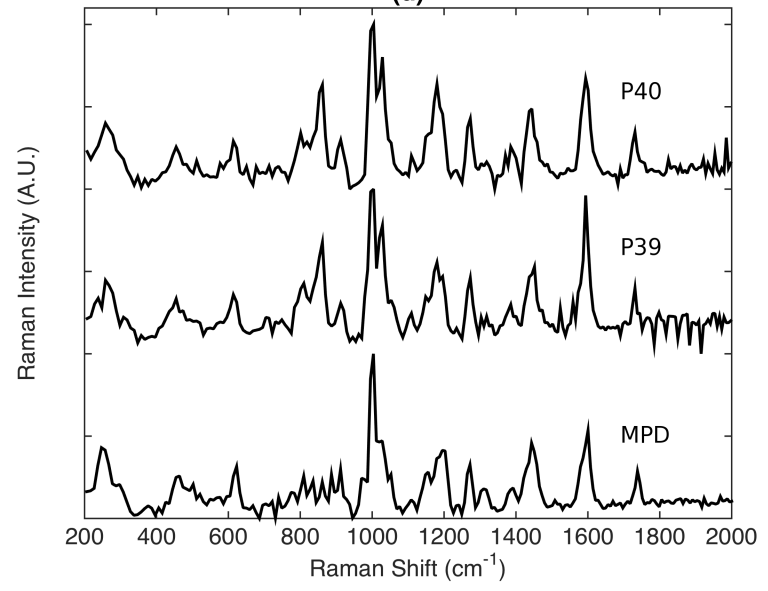

(f)

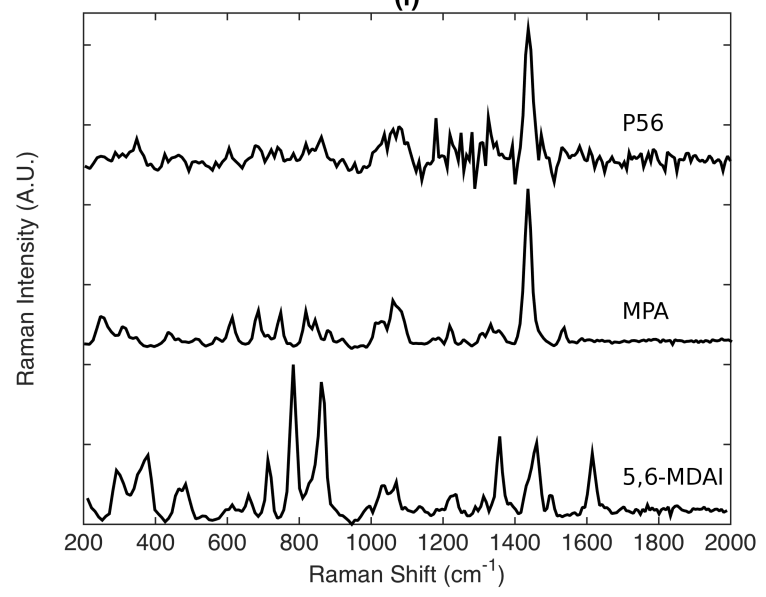

Fig. 3: Raman spectra of selected products and associated reference spectra from the aminoindane (a), arylalkylamine (b), benzodiazepine (c), piperidine \& pyrrolidine (d), other (e), and branded product (f) categories using a $1064 \mathrm{~nm}$ excitation wavelength. Product numbers and reference names are labelled on the spectra. The spectra presented have been normalised to the maximum peak. 


\subsubsection{Benzodiazepines}

Nine benzodiazepine (BZD) samples, purchased from the internet, were analysed using both Raman instruments and GC-MS (Table 2). The products included two different BZDs, etizolam and pyrazolam. Benzodiazepines are pharmaceuticals, which exert depressant, anxiolytic, hypnotic and muscle relaxant effects. Due to the high level of excipients present in the tablet/pellet formulation, it was necessary to remove the coating and increase the concentration of the analysis solution (ca. $45 \mathrm{mg} \mathrm{mL}^{-1}$ ) to enable detection of the NPS via GC-MS. Both etizolam and pyrazolam were then identified in all nine samples using GC-MS. Interestingly, P30 and 32 shared three active ingredients despite being purchased from different websites and having a different appearance, suggesting a similar supply chain. When using the standard $785 \mathrm{~nm}$ laser no correlations to a BZD were found in any of the nine samples even those samples which were confirmed to contain a BZD via GC-MS. Pyrazolam was not present in the Raman libraries but the products were included to investigate analogue selectivity. However, P30, 32 and 33 - 35 correlated to the cutting agents LAC ( $87 \pm 5$ to 91 $\pm 5 \%)$ and MCC $(83 \pm 3$ to $87 \pm 2 \%)$, respectively. High fluorescent backgrounds occurred for four samples (i.e., P27 - 29 and 31), which again resulted in false positive correlations to MPA $(96.9 \pm 0.4$ to $98.5 \pm 0.7 \%)$ as the $1438 \mathrm{~cm}^{-1}$ characteristic peak was not visible in the spectra. Using the $1064 \mathrm{~nm}$ source, no correlations to a BZD resulted for any of the nine samples. However, P30, 32 and 33 - 35 correlated to the same cutting agents as found with the $785 \mathrm{~nm}$ source with similar \% HQIs. Due to reduced fluorescence, P27 - 29 correlated to MCC ( $70 \pm 1$ to $70 \pm 9 \%$ ). For example Figure 2 demonstrates this reduction in fluorescence comparing the two wavelength sources with P27. The subsequent identification of MCC is better illustrated in Figure 3c using P27 and 33 where the MCC signature is clearly visible when comparing it with an MCC reference (c.a., 397, 1094, and $1355 \mathrm{~cm}^{-1}$ ). For P27, the strong signature peak of etizolam at $1496 \mathrm{~cm}^{-1}$ (Figure S1 in Supplementary Information) is clearly not visible. As mentioned above both $\mathrm{MCC}$ and LAC do not readily dissolve in methanol and have low volatility; hence, it is often not detected with GC-MS analysis, but may still be present in the sample. Microcrystalline cellulose (MCC) is a common diluent used in pharmaceutical tablets and LAC is commonly used in direct compression tableting applications and is also used as a tablet filler and binder. As most of the BZD products were in tablet or pellet form, the presence of these excipients in high concentration is likely. Products 27, 28 and 29 were purchased from three different websites (Table S1); however, the three batches, all turquoise in colour, have been shown to contain MCC and etizolam using the $1064 \mathrm{~nm}$ Raman spectrometer and GC-MS, respectively. This may indicate that despite being sold on different websites and compressed with different tablet dies, the powder mix could have come from the same supplier. In summary, no NPS ingredients were identified in the BZD products using the 'first pass' algorithm or by visual inspection of the spectra as the products were largely composed of excipients used for tablet and pellet construction (i.e., MCC and LAC), which was also observed in a recent study using a benchtop Raman spectrometer. ${ }^{[38]}$ This indicates that for these BZDs and perhaps other NPS in tablet form, identification of the active ingredient can be challenging (i.e., resulting in false negatives), as the amount of active ingredient may be significantly lower relative to the excipients. The use of spectral subtraction is a possible tool for mixtures that may reduce 
these types of false negatives; it could be used to improve the identification of NPS with low content in the presence of larger amounts of cutting agents that results in a larger Raman signal than the NPS. ${ }^{[38]}$

Table 2: Results from the analysis of benzodiazepine, piperidine and pyrrolidine, plants and extracts and other internet products using two handheld Raman spectrometers $\left(\lambda_{\mathrm{ex}}=785\right.$ and $\left.1064 \mathrm{~nm}\right)$ and GC-MS ${ }^{1}$

\begin{tabular}{|c|c|c|c|c|c|c|c|c|}
\hline \multirow[t]{2}{*}{$\begin{array}{l}\text { Product } \\
\text { No. }\end{array}$} & \multirow[t]{2}{*}{$\begin{array}{l}\text { Product } \\
\text { name }\end{array}$} & \multicolumn{2}{|c|}{$\begin{array}{c}\text { Handheld Raman } \\
785 \mathrm{~nm} \\
\end{array}$} & \multicolumn{2}{|c|}{$\begin{array}{c}\text { Handheld Raman } \\
1064 \mathrm{~nm}\end{array}$} & \multicolumn{3}{|c|}{ GC-MS } \\
\hline & & ID & $\%$ HQI & ID & $\%$ HQI & $\begin{array}{l}\text { RT } \\
(\mathrm{min})\end{array}$ & $\begin{array}{l}\text { Base } \\
\text { Peak } \\
(\mathrm{m} / \mathrm{z}) \\
\end{array}$ & MS ID \\
\hline \multicolumn{9}{|c|}{ Benzodiazepines } \\
\hline 27 & Etizolam & $\mathrm{MPA}^{3}$ & $96.9 \pm 0.4$ & $\mathrm{MCC}$ & $70 \pm 1$ & 23.1 & 342 & Etizolam \\
\hline 28 & Etizolam & $\mathrm{MPA}^{3}$ & $97.49 \pm 0.05$ & MCC & $70 \pm 4$ & 23.2 & 342 & Etizolam \\
\hline 29 & Etizolam & $\mathrm{MPA}^{3}$ & $96.9 \pm 0.7$ & $\mathrm{MCC}$ & $70 \pm 9$ & 23.2 & 342 & Etizolam \\
\hline 30 & Etizolam & LAC & $87 \pm 5$ & LAC & $80 \pm 7$ & $\begin{array}{l}16.9 \\
19.3 \\
22.9 \\
23.2\end{array}$ & $\begin{array}{l}339 \\
359 \\
270 \\
342\end{array}$ & $\begin{array}{l}\text { JWH-022 } 2 \\
\text { AM-2201 }^{2} \\
1-\mathrm{NI}^{2,4} \\
\text { Etizolam }\end{array}$ \\
\hline 31 & Etizolam & $\mathrm{MPA}^{3}$ & $98.5 \pm 0.7$ & $\begin{array}{l}\text { Phospho- } \\
\text { rous }\end{array}$ & $60 \pm 3$ & 23.1 & 342 & Etizolam \\
\hline 32 & Etizolam & LAC & $91 \pm 5$ & LAC & $88.6 \pm 2.3$ & $\begin{array}{l}16.7 \\
21.8 \\
22.9 \\
23.2\end{array}$ & $\begin{array}{l}339 \\
268 \\
270 \\
342\end{array}$ & $\begin{array}{l}\mathrm{JWH}-022^{2} \\
1-\mathrm{N}-2-\mathrm{MI}^{2,4} \\
1-\mathrm{NI}^{2,4} \\
\text { Etizolam }\end{array}$ \\
\hline 33 & Pyrazolam & $\mathrm{MCC}$ & $86 \pm 2$ & $\mathrm{MCC}$ & $80 \pm 6$ & 13.5 & 353 & Pyrazolam $^{2}$ \\
\hline 34 & Pyrazolam & $\mathrm{MCC}$ & $87 \pm 2$ & $\mathrm{MCC}$ & $90 \pm 2$ & 13.5 & 353 & Pyrazolam $^{2}$ \\
\hline 35 & Pyrazolam & $\mathrm{MCC}$ & $83 \pm 3$ & $\mathrm{MCC}$ & $81 \pm 3$ & 13.5 & 353 & Pyrazolam $^{2}$ \\
\hline \multicolumn{9}{|c|}{ Piperidines \& pyrrolidines } \\
\hline 36 & $\begin{array}{l}\text { Ethyl } \\
\text { phenidate }\end{array}$ & MPD & $64 \pm 4$ & MPD & $76.6 \pm 0.4$ & 14 & 84 & MPD \\
\hline 37 & $\begin{array}{l}\text { Ethyl } \\
\text { phenidate }\end{array}$ & MPD & $65 \pm 2$ & MPD & $76.8 \pm 0.4$ & 14 & 84 & MPD \\
\hline 38 & $\begin{array}{l}\text { Ethyl } \\
\text { phenidate }\end{array}$ & Inconsistent & rrelations & No match & & 19.4 & 359 & AM-2201 2 \\
\hline 39 & $\begin{array}{l}\text { Ethyl } \\
\text { phenidate }\end{array}$ & $\begin{array}{l}\text { Cetyl- } \\
\text { pyridinium } \\
\text { chloride }\end{array}$ & $61 \pm 20$ & MPD & $80 \pm 2$ & 14 & 84 & MPD \\
\hline 40 & $\begin{array}{l}\text { Ethyl } \\
\text { phenidate }\end{array}$ & MPD & $63 \pm 5$ & MPD & $76.6 \pm 0.2$ & 14 & 84 & MPD \\
\hline 41 & $\begin{array}{l}\text { Ethyl } \\
\text { phenidate }\end{array}$ & Inconsistent & rrelations & No match & & 19.4 & 359 & $\mathrm{AM}-2201^{2}$ \\
\hline \multicolumn{9}{|c|}{ Plants and Extracts } \\
\hline 42 & $\begin{array}{l}\text { LSA } \\
\text { Morning } \\
\text { Glory Seeds }\end{array}$ & $\mathrm{MPA}^{3}$ & $96 \pm 2$ & No match & & \multicolumn{2}{|c|}{ No match } & \\
\hline \multicolumn{9}{|c|}{ Other } \\
\hline 43 & DXM & Talc & $69 \pm 3$ & CAF & $80 \pm 10$ & $\begin{array}{l}14.4 \\
14.8\end{array}$ & $\begin{array}{l}194 \\
190\end{array}$ & $\begin{array}{l}\text { CAF } \\
\mathrm{MXE}^{2}\end{array}$ \\
\hline 44 & DXM & Inconsistent & rrelations & DXM & $60 \pm 9$ & 16.6 & 271 & DXM \\
\hline 45 & DXM & DXM & $57 \pm 1$ & DXM & $64.0 \pm 0.4$ & 16.6 & 271 & DXM \\
\hline 46 & DXM & DXM & $59 \pm 6$ & DXM & $63.8 \pm 0.4$ & 16.6 & 271 & DXM \\
\hline 47 & DXM & DXM & $84.1 \pm 0.2$ & DXM & $90 \pm 4$ & 16.6 & 271 & DXM \\
\hline
\end{tabular}

${ }^{1}$ ID: identification; RT: retention time; NC: not confirmed

${ }^{2}$ Raman spectrum of substance not present in both Raman libraries

${ }^{3}$ Suspected false positive

${ }^{4} 1$-NI: 1-Naphthoyl indole; 1-N-2-MI: 1-Naphthoyl-2-methyl indole 


\subsubsection{Piperidines \& pyrrolidines}

Six ethylphenidate (piperidine) samples, purchased from the internet, were analysed using both Raman instruments and GC-MS (Table 2). Ethylphenidate (EPD) is a synthetic analogue of MPD and was encountered at EU level and in the UK for the first time in 2011. ${ }^{[50]}$ The GC-MS analysis confirmed the presence of $\mathrm{MPD}^{[51]}$ in four products (i.e., P36, 37, 39 and 40). Until recently, EPD was uncontrolled in the UK, perhaps a reason why the controlled substance MPD was substituted for EPD in these samples. These products are an example of how NPS may be marketed as 'legal' products, where in fact they contain a controlled drug. Thus, only MPD was added to the Raman libraries as EPD was not identified via GC-MS. Products 38 and 41 were purchased from the same website and a similar active ingredient was identified using GC-MS (i.e., AM-2201). When using the standard $785 \mathrm{~nm}$ laser, P36, 37 and 40 correlated to MPD (63 \pm 5 to $65 \pm 2 \%$ ). Products 38 and 41 showed inconsistent Raman responses due to high fluorescent backgrounds. The Raman spectra of P39 correlated to cetylpyridinium chloride $(61 \pm 20 \%)$ where very weak Raman signals were observed on top of a high fluorescence background (Figure 2a), again suggesting a false positive. Using the $1064 \mathrm{~nm}$ source, four of the six samples (i.e., P36, 37, 39 and 40) correlated to MPD, the analogue of EPD, with a \% HQI of $76.6 \pm 0.2$ to $80 \pm 2$. Figure $2 b$ displays the Raman spectra of P39 after using the longer wavelength reducing fluorescence. The spectra of two products, P39 and 40, compared to the MPD signature shows distinct peaks at 1029, 1180, 1428 and $1588 \mathrm{~cm}^{-1}$ (Figure 3d) which correspond to Raman bands for the MPD signature (Figure S1 in Supplementary Information). Products 38 and 41 resulted in 'no match' using the algorithm, however Raman bands at 511, 668, 775, 1012, 1370, 1516 and $1622 \mathrm{~cm}^{-1}$ corresponded to peaks for the AM-2201 reference spectra (unpublished work). In summary, the $785 \mathrm{~nm}$ source successfully identified the NPS ingredient in three products, while use of the $1064 \mathrm{~nm}$ source reduced fluorescence and improved 'first pass' NPS identification for one of the three remaining products in addition to evidence for the presence of AM-2201 in two products.

\subsubsection{Plants and Extracts}

LSA morning glory seeds (P42) were purchased from the internet and analysed using both Raman instruments and GC-MS. Morning glory seeds are known to be sacred seeds, originally used by some Mexican Indian tribes. They contain lysergic acid amide (LSA), which is the non-alkylated amide analogue of the schedule I controlled lysergic acid diethyl amide (LSD). ${ }^{[52]}$ As these samples were seeds, before analysis they were ground using an agate mortar and pestle. LSA morning glory seeds were characterised using the black shell and grey content. Using GC-MS, the content of the seeds was not identified as no chromatographic peaks were observed. When using the standard $785 \mathrm{~nm}$ laser, P42 correlated to MPA $(96 \pm 2 \%)$ as seen with other products using the $785 \mathrm{~nm}$ laser. However, this is again a potential false positive result as the content was not confirmed using GC-MS. Using the $1064 \mathrm{~nm}$ source, no match was found and the sample was burned upon analysis using all methods. Seed samples such as these, which are dark in colour, are particularly problematic when analysing via Raman even with the $1064 \mathrm{~nm}$ wavelength. 


\subsubsection{Other}

Five DXM samples, purchased from the internet, were analysed using both Raman instruments and GC-MS (Table 2). Dextromethorphan (DXM) has been classified as 'other' in the UNODC (2014) report. ${ }^{[53]}$ DXM is a non-opioid anti-tussive drug and is the d-isomer of the codeine analogue levorphanol. It inhibits the re-uptake of serotonin at therapeutic doses. ${ }^{[54]}$ The GC-MS analysis identified $\mathrm{DXM}^{[55]}$ in four of the five products (i.e., $\mathrm{P} 44-47$ ). When using the standard $785 \mathrm{~nm}$ laser, correlations to DXM were found in three of the five samples (P45 - 47) with \% HQIs ranging from $57 \pm 1$ to $84.1 \pm 0.2 \%$. Product 43 correlated to TAL $(69 \pm 3 \%$ ) (Figure $2 \mathrm{a}$ ) and P44 resulted in inconsistent correlations; these spectra showed Raman signals on top of slightly fluorescent backgrounds. Using the $1064 \mathrm{~nm}$ source, four of the five samples (i.e., P44 - 47) correlated to the NPS on the label (DXM) with an HQI of $60 \pm 9$ to $90 \pm 4 \%$. A reduction in fluorescence was most noticeable for P43 (Figure $2 b)$ which correlated to the unclaimed adulterant CAF at a \% HQI of $80 \pm 10 \%$. Figure $3 \mathrm{e}$ shows an example of two products' spectra, P43 and 47, and their similarity to the highest correlation signature spectrum. The spectra for P43 and CAF are very similar with notable peaks at 549, 1325, 1600 and $1690 \mathrm{~cm}^{-1}$ even though MXE was also identified using GC-MS; and the spectra for P47 and DXM are also very similar with notable peaks at $686,852,1242$, and $1436 \mathrm{~cm}^{-1}$. In summary, the $785 \mathrm{~nm}$ source successfully identified the NPS ingredient in three products, while use of the $1064 \mathrm{~nm}$ source reduced fluorescence and improved NPS identification for one of the two remaining products using the 'first pass' identification and visual inspection of the spectra

\subsubsection{Branded products}

Thirteen branded products, purchased from the internet, were analysed using both Raman instruments and GC-MS (Table 3). Internet products are often branded with names such as blast, bliss, bloom and blow. ${ }^{[35]}$ Although these products are marketed with brand names, most branded products in this study did have a label claim stating ingredients except for P48 and 59. The analysis using GC-MS identified seven different NPS substances (Table 3). Interestingly, both P23 labelled as MPA and P59 labelled as Route 56 were purchased from the same website and shared four active ingredients (i.e., MPA, 6-APB, CAF and 5-MeODALT), again suggesting a similar supply chain. When using the standard $785 \mathrm{~nm}$ laser, 'first pass' correlations to a NPS substance were found for 8 out of 13 samples, even though all but one sample was confirmed to contain an NPS via GC-MS. However, for seven of these products (i.e., P49 - 55 and 57 - 60) the spectra showed very high fluorescent backgrounds resulting in either a false positive correlation to MPA $(92.5 \pm 0.4$ to $99.1 \pm 0.1 \%)$ (e.g., see Figure 2a for spectrum of P53) or an inconsistent correlation. The calculation of the HQI algorithm has been shown to be affected by background fluorescence in unknown spectra, depicting slope and offset as differences from library spectra. ${ }^{[47]}$ Pink panthers (P56) correlated to 5,6-MDAI with a \% HQI of $80 \pm 7$, whereas the product Blow (P48) correlated to BEN with a $\%$ HQI of $76 \pm 10$. Using the $1064 \mathrm{~nm}$ source, two additional NPS, MPA (80 $\pm 2 \%)$ and delta 9-THC (59.3 $\pm 0.8 \%$, were identified in P56 and 60. The presence of MPA was confirmed using GC-MS, however delta 9-THC could potentially be a false positive result, since 9-THC was not identified by the MS libraries. Figure $2 \mathrm{~b}$ shows the improved 
Raman spectra of P53 after using the $1064 \mathrm{~nm}$ source where clear distinct Raman bands are visible. The improved Raman spectra still resulted in 'no match' using the algorithm, however characteristic peaks for CAF (e.g., 549 and $1322 \mathrm{~cm}^{-1}$ ) and 2-AI (e.g., 775, 844, and $1030 \mathrm{~cm}^{-1}$ ) were clearly visible. Jones et al. evaluated Raman spectra of NPS mixtures by subtracting the spectra of pure substances sequentially after identifying the substances using a Raman microscope. ${ }^{[38]}$ Interestingly, the product Pink panthers (P56), which was confirmed to contain both 5,6-MDAI and MPA with GC-MS analysis correlated to 5,6-MDAI using the $785 \mathrm{~nm}$ source and to MPA using the $1064 \mathrm{~nm}$ source. This may be the result of mixture heterogeneity inherent to branded products, despite efforts to vortex mix. Figure $3 \mathrm{f}$ shows peaks in the Raman spectrum for P56 corresponding to those of primarily MPA, i.e., 595, $677,810,1052$, and $1436 \mathrm{~cm}^{-1}$; peaks corresponding to 5,6-MDAI are not observed. In summary, the 'first pass' identification of branded NPS products was very challenging using both handheld Raman instruments resulting in many inconsistent correlations, 'no match' founds, and false positive matches to MPA. This was due mainly to high fluorescent signals using the $785 \mathrm{~nm}$, which was significantly reduced using the $1064 \mathrm{~nm}$ source, and the chemical complexity of the samples. A mixture algorithm or spectral subtraction would be useful for these types of samples to improve 'first pass' identification as peaks were identified which were consistent with NPS reference spectra. 
Table 3: Results from the analysis of branded internet products using two handheld Raman spectrometers $\left(\lambda_{\mathrm{ex}}=\right.$ 785 and $1064 \mathrm{~nm}$ ) and GC-MS ${ }^{1}$

\begin{tabular}{|c|c|c|c|c|c|c|c|c|}
\hline \multirow[t]{2}{*}{$\begin{array}{l}\text { Product } \\
\text { No. }\end{array}$} & \multirow[t]{2}{*}{$\begin{array}{l}\text { Product } \\
\text { name }\end{array}$} & \multicolumn{2}{|c|}{$\begin{array}{c}\text { Handheld Raman } \\
785 \mathrm{~nm} \\
\end{array}$} & \multicolumn{2}{|c|}{$\begin{array}{c}\text { Handheld Raman } \\
1064 \mathrm{~nm}\end{array}$} & \multicolumn{3}{|c|}{ GC-MS } \\
\hline & & ID & $\%$ HQI & ID & $\%$ HQI & $\begin{array}{l}\text { RT } \\
\text { (min) }\end{array}$ & $\begin{array}{l}\text { Base } \\
\text { Peak } \\
(\mathrm{m} / \mathrm{z}) \\
\end{array}$ & MS ID \\
\hline \multicolumn{9}{|c|}{ Branded products } \\
\hline 48 & Blow & BEN & $76 \pm 10$ & BEN & $90 \pm 7$ & $\begin{array}{l}12.4 \\
14.1\end{array}$ & $\begin{array}{l}120 \\
84\end{array}$ & $\begin{array}{l}\text { BEN } \\
\text { MPD }\end{array}$ \\
\hline 49 & Blurberry & $\mathrm{MPA}^{3}$ & $99.1 \pm 0.1$ & No match & & $\begin{array}{l}9.5 \\
14.4\end{array}$ & $\begin{array}{l}133 \\
194\end{array}$ & $\begin{array}{l}2-\mathrm{AI} \\
\mathrm{CAF}\end{array}$ \\
\hline 50 & High beams & $\mathrm{MPA}^{3}$ & $97.7 \pm 0.1$ & No match & & $\begin{array}{l}9.4 \\
14.4\end{array}$ & $\begin{array}{l}133 \\
194\end{array}$ & $\begin{array}{l}\text { 2-AI } \\
\text { CAF }\end{array}$ \\
\hline 51 & Magic Beans & $\mathrm{MPA}^{3}$ & $92.5 \pm 0.4$ & Inconsister & orrelations & $\begin{array}{l}9.4 \\
14.4\end{array}$ & $\begin{array}{l}133 \\
194\end{array}$ & $\begin{array}{l}\text { 2-AI } \\
\text { CAF }\end{array}$ \\
\hline 52 & $\begin{array}{l}\text { Pink } \\
\text { Champagnes }\end{array}$ & $\mathrm{MPA}^{3}$ & $98.0 \pm 0.4$ & Inconsister & orrelations & $\begin{array}{l}9.4 \\
14.4\end{array}$ & $\begin{array}{l}133 \\
194\end{array}$ & $\begin{array}{l}2-\mathrm{AI} \\
\mathrm{CAF}\end{array}$ \\
\hline 53 & $\begin{array}{l}\text { Pink } \\
\text { Champagnes }\end{array}$ & $\mathrm{MPA}^{3}$ & $98.4 \pm 0.2$ & No match & & $\begin{array}{l}9.2 \\
14.4\end{array}$ & $\begin{array}{l}133 \\
194\end{array}$ & $\begin{array}{l}2-\mathrm{AI} \\
\mathrm{CAF}\end{array}$ \\
\hline 54 & $\begin{array}{l}\text { Pink } \\
\text { Champagnes }\end{array}$ & $\mathrm{MPA}^{3}$ & $98.4 \pm 0.1$ & CAF & $54.2 \pm 0.2$ & $\begin{array}{l}9.4 \\
14.4\end{array}$ & $\begin{array}{l}133 \\
194\end{array}$ & $\begin{array}{l}2-\mathrm{AI} \\
\mathrm{CAF}\end{array}$ \\
\hline 55 & $\begin{array}{l}\text { Pink } \\
\text { Champagnes }\end{array}$ & $\mathrm{MPA}^{3}$ & $98.3 \pm 0.2$ & $\begin{array}{l}\text { Phospho- } \\
\text { rous }\end{array}$ & $70 \pm 3$ & $\begin{array}{l}9.3 \\
14.5\end{array}$ & $\begin{array}{l}133 \\
194\end{array}$ & $\begin{array}{l}2-\mathrm{AI} \\
\mathrm{CAF}\end{array}$ \\
\hline 56 & Pink panthers & 5,6-MDAI & $80 \pm 7$ & MPA & $80 \pm 2$ & $\begin{array}{l}8.9 \\
12.7\end{array}$ & $\begin{array}{l}58 \\
160\end{array}$ & $\begin{array}{l}\text { MPA } \\
5,6-\mathrm{MDAI}\end{array}$ \\
\hline 57 & Punk plus & Inconsisten & orrelations & L-TYR & $54.7 \pm 0.7$ & $\begin{array}{l}10.7 \\
14.4\end{array}$ & $\begin{array}{l}106 \\
194\end{array}$ & $\begin{array}{l}\text { NIA } \\
\text { CAF }\end{array}$ \\
\hline 58 & Recovery & Inconsisten & orrelations & Data acqui & on failed & 7.1 & 71 & No match \\
\hline 59 & Route 56 & Inconsisten & orrelations & MCC & $60 \pm 6$ & $\begin{array}{l}8.8 \\
11.6 \\
14.0 \\
14.4 \\
17.3\end{array}$ & $\begin{array}{l}58 \\
44 \\
84 \\
194 \\
110\end{array}$ & $\begin{array}{l}\text { MPA } \\
6-\mathrm{APB} \\
\mathrm{MPD} \\
\mathrm{CAF} \\
5-\mathrm{MeO}- \\
\text { DALT }^{2}\end{array}$ \\
\hline 60 & White Pearls & Inconsisten & orrelations & $\begin{array}{l}\text { Delta 9- } \\
\text { THC }\end{array}$ & $59.3 \pm 0.8$ & $\begin{array}{l}12.3 \\
14.4\end{array}$ & $\begin{array}{l}44 \\
194\end{array}$ & $\begin{array}{l}\text { 5-APDB }{ }^{2} \\
\text { CAF }\end{array}$ \\
\hline
\end{tabular}

${ }^{1}$ ID: identification; RT: retention time; NC: not confirmed

${ }^{2}$ Raman spectrum of substance not present in both Raman libraries

${ }^{3}$ Suspected false positive

\section{Conclusion}

In this study, handheld Raman spectroscopy with two excitation sources was used to identify NPS in internet products using a 'first pass' matching algorithm as well as visual inspection of Raman spectra. The 'first pass' algorithm approach successfully identified an NPS in 29 out of $60(48 \%)$ diverse and chemically complex internet products using a $1064 \mathrm{~nm}$ laser source. An overview of the results is presented in Table 4. An increase in the laser excitation wavelength from 785 to $1064 \mathrm{~nm}$ improved positive NPS 'first pass' identification (i.e., from 11 to 29 substances). Visual inspection of the spectra indicated that these improvements were mainly the result of reduced fluorescence, most likely originating from cutting agents and coloured constituents composing the products. Correlations between the internet products with the NPS signatures, that were confirmed with GC-MS, ranged from 57.0 to $84.1 \% \mathrm{HQI}$ with typical RSDs $<10 \%$ using the $785 \mathrm{~nm}$ source and from 60.0 to $91.3 \%$ HQI with typical 
RSDs $<7 \%$ using the $1064 \mathrm{~nm}$ source. Thus, reduced matching algorithm thresholds may be required when monitoring NPS products in the field. A higher number of false positives and false negative were observed when using the $785 \mathrm{~nm}$ source, again resulting mainly from the dominant fluorescent background with no notable Raman bands produced by these samples. False negatives observed for both wavelength sources were also due to low NPS concentration and/or high chemical complexity of the product. For example, no etizolam Raman bands were observed for the tablet and pellets as they were largely composed of common excipients with a relatively low etizolam concentration. Chemically complex samples, such as some of the 'branded products', did not correlate to an NPS signature using the 'first pass' identification but did showed marked improvement in the Raman spectra upon using the $1064 \mathrm{~nm}$ source where characteristic Raman bands of the references were observed. In these cases spectral subtraction could be used to further assist identification. Handheld Raman spectroscopy employing a $1064 \mathrm{~nm}$ laser source has shown promise for the chemical identification of NPS products in the field; in particular, for NPS samples that are highly fluorescent. Future work employing a $1064 \mathrm{~nm}$ source should focus on further parameter optimisation, spectral processing and investigating mixture algorithms with improved NPS libraries.

Table 4: A summary of the results obtained for NPS identification using the handheld Raman spectrometers $\left(\lambda_{\text {ex }}=785 \text { and } 1064 \mathrm{~nm}\right)^{1}$

\begin{tabular}{lcc}
\hline Category & $\boldsymbol{\lambda}_{\text {ex }} \mathbf{7 8 5} \mathbf{~ n m}$ & $\boldsymbol{\lambda}_{\text {ex }} \mathbf{1 0 6 4} \mathbf{~ n m}$ \\
\hline Identification of NPS & 11 & 29 \\
Identification of adulterant & 2 & 4 \\
Identification of cutting agent $^{2}$ & 7 & 14 \\
Fluorescence & 38 & 0 \\
Inconsistent correlation $^{2}$ & 10 & 2 \\
No match & 2 & 7 \\
False positive for an NPS & 28 & 1 \\
False negative for an NPS & 46 & 28 \\
\hline
\end{tabular}

${ }^{1}$ Raman spectrometers were used with set parameters as stated in the method section

${ }^{2}$ Cutting agents were not confirmed via GC-MS 


\section{References}

[1] UNODC. United Nation Office on Drugs and Crime. The challenge of new psychoactive substances, United Nations Office on Drugs and Crime, 2013

[2] ACMD. Advisory Council on the Misuse of Drugs. Consideration of the Novel Psychoactive Substances ('Legal Highs'), United Kingdom, 2011

[3] Psychoactive substances Act 2016, UK Parliament, 2016

[4] M. Evans-Brown, Gallegos, A, Francis, W., Christie, R., Cunningham, A., Sekula, J., Almeida, A. \& Sedefov, R. New psychoactive substances in Europe. An update from the EU Early Warning System, European Monitoring Centre for Drugs and Drug Addiction (EMCDDA), Lisbon, 2015

[5] EMCDDA-Europol. EMCDDA-Europol 2014 Annual Report on the implementation of Council Decision 2005/387/JHA, Luxembourg, 2015

[6] L. A. King and R. Sedefov. Early-warning system on new psychoactive substances. Operating Guidelines., EMCDDA - Europol, Luxembourg, 2007

[7] D. Mackie. Regulatory Impact Statement. Amendment to the Psychoactive Substance Act 2013, New Zealand, 2013

[8] L. Morrison. Notice of meeting and agenda. Health, social care and housing committee, The City of Edinburgh Council, Edinburgh, 2015

[9] D. M. Wood and P. I. Dargan. Novel psychoactive substances: How to understand the acute toxicity associated with the use of these substances. Therapeutic Drug Monitoring 2012, 34, 363. [10] H. Regunath, V. K. Ariyamuthu, P. Dalal and M. Misra. Bath salt intoxication causing acute kidney injury requiring hemodialysis. Hemodialysis International 2012, 16, S47.

[11] J. M. Corkery, F. Schifano and A. H. Ghodse in Mephedrone-Related Fatalities in the United Kingdom: Contextual, Clinical and Practical Issues, Pharmacology, Vol. (Ed. L. Gallelli), 2012.

[12] C. O'Neill. Novel Psychoactive Substances: risks and harms. Community Practitioner 2014, 87, 45.

[13] A. Pourmand, P. Armstrong, M. Mazer-Amirshahi and H. Shokoohi. The evolving high New designer drugs of abuse. Human \& Experimental Toxicology 2014, 0960327113514100.

[14] M. P. Elie, L. E. Elie and M. G. Baron. Keeping pace with NPS releases: fast GC-MS screening of legal high products. Drug Testing and Analysis 2013, 5, 281.

[15] L. A. Reitzel, P. W. Dalsgaard, I. B. Müller and C. Cornett. Identification of ten new designer drugs by GC-MS, UPLC-QTOF-MS, and NMR as part of a police investigation of a Danish Internet company. Drug Testing and Analysis 2012, 4, 342.

[16] J. P. Smith, J. P. Metters, C. Irving, O. B. Sutcliffe and C. E. Banks. Forensic electrochemistry: the electroanalytical sensing of synthetic cathinone-derivatives and their accompanying adulterants in "legal high" products. Analyst 2014.

[17] F. T. Peters and M. R. Meyer, 'Analytical techniques for the detection of novel psychoactive substances and their metabolites' in Novel psychoactive substances: classification, pharmacology and toxicology, Elsevier Science, Burlington, 2013.

[18] S. D. Brandt, P. F. Daley and N. V. Cozzi. Analytical characterization of three trifluoromethylsubstituted methcathinone isomers. Drug Testing and Analysis 2012, 4, 525.

[19] J. F. Casale and P. A. Hays. The characterization of 4-and 5-iodo-2-aminoindan. Microgram Journal 2012, 9, 18.

[20] J. F. Casale and P. A. Hays. Characterization of the "methylenedioxy-2-aminoindans". Microgram Journal 2011, 8, 43.

[21] M. Baron, M. Elie and L. Elie. An analysis of legal highs-do they contain what it says on the tin? Drug Testing and Analysis 2011, 3, 576.

[22] V. M. R. Zancajo, J. Brito, M. P. Carrasco, M. R. Bronze, R. Moreira and A. Lopes. Analytical profiles of "legal highs" containing cathinones available in the area of Lisbon, Portugal. Forensic Science International 2014, 244, 102. 
[23] E. Fornal. Identification of substituted cathinones: 3, 4-methylenodioxy derivatives by high performance liquid chromatography-quadrupole time of flight mass spectrometry. Journal of Pharmaceutical and Biomedical Analysis 2013.

[24] S. Dresen, S. Kneisel, W. Weinmann, R. Zimmermann and V. Auwärter. Development and validation of a liquid chromatography-tandem mass spectrometry method for the quantitation of synthetic cannabinoids of the aminoalkylindole type and methanandamide in serum and its application to forensic samples. Journal of Mass Spectrometry 2011, 46, 163.

[25] M. D. Hargreaves. Drugs of Abuse-Application of Handheld FT-IR and Raman Spectrometers. Infrared and Raman Spectroscopy in Forensic Science 2012, 339.

[26] P. Kavanagh, J. O'Brien, J. Fox, C. O'Donnell, R. Christie, J. D. Power and S. D. McDermott. The analysis of substituted cathinones. Part 3. Synthesis and characterisation of 2,3-methylenedioxy substituted cathinones. Forensic Science International 2012, 216, 19.

[27] K. Tsujikawa, T. Yamamuro, K. Kuwayama, T. Kanamori, Y. T. Iwata, K. Miyamoto, F. Kasuya and $\mathrm{H}$. Inoue. Application of a portable near infrared spectrometer for presumptive identification of psychoactive drugs. Forensic Science International 2014, 242, 162.

[28] R. Risoluti, S. Materazzi, A. Gregori and L. Ripani. Early detection of emerging street drugs by near infrared spectroscopy and chemometrics. Talanta.

[29] SWGDRUG. Scientific Working Group for the Analysis of Seized Drugs (SWGDRUG)

Recommendations, USA, 2014

[30] J. M. Chalmers, H. G. M. Edwards and M. D. Hargreaves in Infrared and Raman Spectroscopy in Forensic Science, Wiley, Hoboken, 2011.

[31] A. Assi, A. Guirguis, H. Sheelagh, S. Fergus and J. Stair. Analysis of 'legal high' substances and common adulterants using handheld spectroscopic techniques. Analytical Methods 2015, 7, 736.

[32] S. P. Stewart, S. E. J. Bell, N. C. Fletcher, S. Bouazzaoui, Y. C. Ho, S. J. Speers and K. L. Peters.

Raman spectroscopy for forensic examination of $\beta$-ketophenethylamine " legal highs": Reference and seized samples of cathinone derivatives. Analytica Chimica Acta 2012, 711, 1.

[33] A. G. Ryder, G. M. O'Connor and T. J. Glynn. Identification and quantitative measurements of narcotics in solid mixtures using near-IR Raman spectroscopy and multivariate analysis. Journal of Forensic Sciences 1999, 44, 1013.

[34] C. R. Maheux and C. R. Copeland. Chemical analysis of two new designer drugs: buphedrone and pentedrone. Drug Testing and Analysis 2011, 4, 17.

[35] R. Christie, E. Horan, J. Fox, C. O'Donnell, H. Byrne, S. McDermott, J. Power and P. Kavanagh.

Discrimination of cathinone regioisomers, sold as 'legal highs', by Raman spectroscopy. Drug Testing and Analysis 2013.

[36] S. Mabbott, E. Correa, D. P. Cowcher, J. W. Allwood and R. Goodacre. Optimization of Parameters for the Quantitative Surface-Enhanced Raman Scattering Detection of Mephedrone Using a Fractional Factorial Design and a Portable Raman Spectrometer. Analytical Chemistry 2012, 85, 923.

[37] S. Mabbott, A. Eckmann, C. Casiraghib and R. Goodacre. $2 p$ or not $2 p$ : tuppence-based SERS for the detection of illicit materialst. Analyst 2013, 138, 118.

[38] L. E. Jones, A. Stewart, K. L. Peters, M. McNaul, S. J. Speers, N. C. Fletcherd and S. E. J. Bell. Infrared and Raman screening of seized novel psychoactive substances: a large scale study of $>200$ samples. Analyst 2016, 141, 902.

[39] S. E. Bell, S. P. Stewart and S. J. Speers, Raman Spectroscopy of Drugs of Abuse, John Wiley \& Sons, Ltd., UK, 2012.

[40] M. D. Hargreaves, A. D. Burnett, T. Munshi, J. E. Cunningham, E. H. Linfield, A. G. Davies and H. G. Edwards. Comparison of near infrared laser excitation wavelengths and its influence on the interrogation of seized drugs-of-abuse by Raman spectroscopy. Journal of Raman Spectroscopy 2009, 40, 1974.

[41] B. Jaganathan and G. P. Singh. Vibrational spectroscopic studies on drugs. World Journal of Pharmacy and Pharmaceutical Sciences 2013, 2, 3467. 
[42] K. A. Bakeev and R. V. Chimenti. Pros and cons of using correlation versus multivariate algorithms for material identification via handheld spectroscopy. European Pharmaceutical Review 2013, 1.

[43] S. Assi, M. D. Osselton and B. Wallis. The evaluation of dual laser handheld Raman spectroscopy for identifying novel psychoactive substances. American Pharmaceutical Review 2016, 1.

[44] A. Gallegos, Novel Psychoactive Substances (Rome) 2014.

[45] P. Sainsbury, A. Kicman, R. Archer, L. King and R. Braithwaite. Aminoindanes-the next wave of 'legal highs'? Drug testing and analysis 2011, 3, 479.

[46] J. F. Casale and P. A. Hays. Methiopropamine: An analytical profile. Microgram Journal 2011, 8, 53.

[47] H. M. Cartwright and M. Sztandera in Soft Computing Approaches in Chemistry Vol. SpringerVerlag Berlin Heidelberg 2003, Germany, 2003, p. 207.

[48] DrugWatch in Drug watch information sheet - MPA, Drug Watch, UK, 2014, pp. 1-6.

[49] J. Casale and P. Hays. The Characterization of 5-and 6-(2-Aminopropyl) 2, 3-dihydrobenzofuran. Microgram Journal 2011, 8, 62.

[50] H. Office in Annual report on the Home Office Forensic Early Warning System (FEWS). A system to identify new psychoactive substances in the UK, Home Office, UK, 2015.

[51] F. Schifano, G. D. Papanti, L. Orsolini and J. Corkery. The consequences of drug misuse on postmarketing surveillance. Expert Review of Clinical Pharmacology 2016, 1.

[52] H. B. Klinke, I. B. Müller, S. Steffenrud and R. Dahl-Sørensen. Two cases of lysergamide intoxication by ingestion of seeds from Hawaiian Baby Woodrose. Forensic Science International 2010, 197, e1.

[53] UNODC. United Nations Office on Drugs and Crime. Global Synthetic Drugs Assessment. Amphetamine-type stimulants and new psychoactive substances, United Nations Office on Drugs and Crime, Vienna, 2014

[54] T. M. Woo and J. R. Hanley. "How High Do They Look?": Identification and Treatment of Common Ingestions in Adolescents. Journal of Pediatric Health Care 2013, 27, 135.

[55] Y. J. Wu, Y. Y. Cheng, S. Zeng and M. M. Ma. Determination of dextromethorphan and its metabolite dextrorphan in human urine by capillary gas chromatography without derivatization. Journal of Chromatography B 2003, 784, 219. 